\title{
Quaternary Trimethyl Chitosan Chloride Capped Bismuth Nanoparticles with Positive Surface Charges: Catalytic and Antibacterial Activities
}

\author{
Yakubu Adekunle Alli ${ }^{1,3} \cdot$ Sheriff Adewuyi ${ }^{1} \cdot{\text { Babatunde Saheed } \text { Bada }^{2} \cdot \text { Sabu Thomas }}^{3} \cdot$ Hazleen Anuar $^{4}$
}

Received: 12 June 2021 / Accepted: 29 July 2021

(c) The Author(s), under exclusive licence to Springer Science+Business Media, LLC, part of Springer Nature 2021

\begin{abstract}
Quaternary trimethyl chitosan-stabilized bismuth nanoparticles (QTMC-BiNPs) with positive surface charges were uniquely synthesized and fully characterized. In the synthesis, Quaternary Trimethyl Chitosan (QTMC), a water-soluble derivative of chitosan (CTS) was prepared using two-step reductive methylation. The new biopolymeric functionalized ligand was further used as capping agent for the synthesis of QTMC-BiNPs which was applied as antibacterial and catalytic agents. The reaction was carried out at room temperature without the use of energy consuming or high-cost instruments. The QTMC and nanocomposites were characterized by proton nuclear magnetic resonance ( ${ }^{1} \mathrm{H}$ NMR), attenuated total reflection Fourier-transform infrared, UV-visible, X-ray diffraction, X-ray photoelectron spectroscopy and energy dispersive X-ray spectroscopic techniques. The topology and morphology of the composites were examined with scanning electron microscopy and high-resolution transmission electron microscopy. Thermogravimetric and differential thermal gravimetric analysis were also conducted. The degree of quaternization and degree of dimethylation values of 63.33 and $11.75 \%$, respectively obtained for QTMC confirmed that the main product is a quaternary derivative. The average particle size of QTMC-BiNPs was evaluated to be between 30 and $45 \mathrm{~nm}$. The QTMC-BiNPs revealed clear and uniform lattice fringes with an estimated interplanar d-spacing of $0.32 \mathrm{~nm}$ confirming the formation of highly crystalline nanocomposites. A further insight into the antibacterial activities of this nanomaterial were carefully examined on Escherichia coli (E. coli) and Staphylococcus aureus (S. aureus) using resazurin based microdilution method for Minimum Inhibitory Concentration (MIC) and Minimum Bactericidal Concentration (MBC). The obtained results revealed that both bacteria pathogens were effectively inhibited/killed by the QTMC-BiNPs at very low concentrations. The MIC of 15.63 and $125 \mu \mathrm{g} / \mathrm{mL}$ were recorded against E. coli and $S$. aureus, respectively while the $\mathrm{MBC}$ of 31.25 and $500.00 \mu \mathrm{g} / \mathrm{mL}$ were estimated against E. coli and S. aureus, respectively. An extensive evaluation of the catalytic capability of the nanocomposites towards the reduction of 4-nitrophenol to 4-aminophenol was also carried out with highly promising result.
\end{abstract}

Keywords Chitosan · Quaternary trimethyl chitosan · Bismuth nanoparticles · Antibacterial activity $\cdot$ Catalytic activity

Sheriff Adewuyi

adewuyis@funaab.edu.ng

1 Department of Chemistry, Federal University of Agriculture Abeokuta, Abeokuta 2240, Ogun State, Nigeria

2 Department of Environmental Management and Toxicology, Federal University of Agriculture Abeokuta, Abeokuta 2240, Ogun State, Nigeria
3 School of Energy Materials and International and Inter University Centre for Nanoscience and Nanotechnology (IIUCNN), Mahatma Gandhi University, Kottayam, Kerala 686560, India

4 Department of Manufacturing and Materials Engineering, Kulliyyah of Engineering, International Islamic University Malaysia, Jalan Gombak, 53100 Kuala Lumpur, Malaysia 


\section{Introduction}

The applications of CTS, a cationic biopolymer of glucosamine and $\mathrm{N}$-acetyl glucosamine, suffer severe limitations since it is insoluble in neutral or alkaline media. Several derivatives of CTS have been reported to overcome the solubility issues encountered in native CTS. The addition of quaternary ammonium halide salt to the CTS structure is a typical example [1-3], which results in a polysaccharide derivative that enhances the properties of the biopolymer. QTMC is a quaternized water friendly derivative of CTS that overcomes the solubility issues presented by ordinary CTS [3, 4]. This quaternized derivative of CTS is positively charged in nature and is soluble across a wide $\mathrm{pH}$ range $[4,5]$. The drug delivery, permeability enhancement, mucoadhesion, and antimicrobial characteristics of QTMC, a derivative of cationic polymer loaded with positive charge, are all improved [5-7]. In general, the use of bio-polymers as capping agents for the synthesis of metal nanoparticles (MNPs) has garnered growing scientific attention due to their biodegradability, accessibility, affordability, biocompatibility and low toxicity. [8]. Thus, the choice of the QTMC as stabilizer for MNPs is because of its ability to interact with metals, low toxicity, and high solubility in neutral solution which makes a perfect candidate for the synthesis of MNPs.

Bismuth is a semimetal with unique electrical characteristics due to its highly anisotropic Fermi level, low carrier composition, long charge channel means free path and tiny effective mass [9]. In recent time, bismuth nanoparticles (BiNPs) have attracted many scientific studies because at the nanoscale region $(1-100 \mathrm{~nm})$, It has intriguing photocatalytic and catalytic characteristics, the former owing to UV absorption caused by its plasmon resonance [10, 11]. Its application in X-ray radiation therapy such as in the treatment of cancer, bacterial infection and in thermoelectricity has also attracted major interest. BiNPs were previously reviewed as effective photocatalysts for water previously [12].

Different methods have been employed in the synthesis of BiNPs [11, 13-15]. Among these, the solution phase chemical method is widely used due to its simplicity. In general, it entails reducing the appropriate metal salt with a reducing agent in the presence of a capping agent which acts as a structure-controlling surfactant [16]. BiNPs are often made in an organic solvent, which raises environmental risks owing to the discharge of hazardous chemicals. Xia and co-workers prepared bismuth nanoparticles and compared the stabilizing efficacy of starch and polyvinylpyrrolidone (PVP) in which starch proves to be more promising than PVP [11].
In another study, BiNPs was prepared by reducing ammonium bismuth citrate, using ethylene glycol as its solvent in the presence of PVP [17]. BiNPs are rarely prepared in aqueous solutions because bismuth salt is hydrophobic, and the produced BiNPs is readily hydrolyzed and oxidized in water and as a result, stabilizing agents are employed to ensure the steady dispersion of BiNPs and to avoid aggregation [11]. Recently, Wang's group revealed a straightforward technique for producing BiNPs using a well-developed ball-milling process, which is a promising way for synthesizing BiNPs [18]. In addition, Gomez and co-workers synthesized BiNPs by flow chemistry under sonication [19]. In furtherance of this effort, owing to the improved properties of QTMC, especially its hydrophilicity and high metal ion interaction, it is hypothesized that this highly charged biopolymer can better serve as a stabilizing agent for BiNPs in an aqueous media.

Consequently, it is opined that the composite of BiNPs can produce a synergistic effect of improved antibacterial properties against gram-negative and gram-positive bacteria. Furthermore, BiNPs have been shown to have activity against a wide range of pathogen microorganism [20]. In addition, BiNPs' catalytic activity has not been well investigated, particularly for the conversion of 4-nitrophenol (4-NP) to 4-aminophenol (4-AP), which is often catalyzed by noble metal nanoparticles such as copper, gold, silver, palladium, and platinum. [11, 17, 21]. The impact of positively charged QTMC-BiNPs on E. coli and $\mathrm{S}$. aureus, as well as the catalytic activity of these nanocomposites for the reduction of 4-NP to 4-AP in aqueous solution under moderate conditions, were focused in this work. The QTMC-BiNPs were synthesized via chemical process and the effect of initial concentration of QTMC saccharide unit on the formation QTMC-BiNPs was investigated. Both the physical and chemical properties of QTMC and QTMC-BiNPs were monitored by attenuated total reflection fourier-transform infrared spectroscopy (ATR-FTIR), thermogravimetry analysis and differential thermogravimetry (TGA/DTG), ultraviolet-vis (UV-vis) spectroscopy, X-ray diffraction (XRD), energy dispersive X-ray (EDX) spectroscopy, scanning electron microscopy (SEM) and high-resolution transmission electron microscopy (HR-TEM). For MIC and MBC, the antibacterial activity of QTMC-BiNPs against gram-negative and gram-positive bacteria was evaluated using the resazurin broth microdilution technique. QTMC-BiNPs was also used to assess the catalytic reduction of 4-NP to 4-AP. The results obtained are herein presented. 


\section{Materials and Method}

\section{Materials}

CTS with a high molecular weight (degree of deacetylation;75\%), N-methyl-2-pyrrolidinone, sodium iodide, and iodo methane were bought analytically from Sigma Aldrich (St. Louis, USA) and utilized without additional purification. Other reagents such as ammonium bismuth citrate (ABC), sodium borohydride $\left(\mathrm{NaBH}_{4}\right)$, sodium hydroxide, ethanol, diethyl ether, sodium chloride, double distilled water, 4-nitrophenol and resazurin salt were all procured from HiMedia Laboratories Pvt., Ltd. (Mumbai-400086, India).

\section{Method of Synthesis of QTMC}

The two-step methylation technique of Chang and colleagues [2] was modified for the synthesis of QTMC, which required reductive methylation of CTS in the presence of a strong base using iodo methane (CH3I, a methylating agent) (Scheme 1). In a typical preparation, $2.0 \mathrm{~g}$ of CTS and $4.8 \mathrm{~g}$ of sodium iodide as a catalyst were dissolved in $80.0 \mathrm{~mL}$ of $\mathrm{N}$-methyl-2-pyrrolidinone (NMP) as base in a $500 \mathrm{~mL}$ double-necked round bottom flask set on a constant temperature water bath at $60{ }^{\circ} \mathrm{C}$ and stirred continuously until full dissolution of CTS was detected. The flask was then attached to a condensation column with running water, and $11.0 \mathrm{~mL}$ of $15 \%(\mathrm{w} / \mathrm{v})$ aqueous $\mathrm{NaOH}$ solution was added to the flask, followed by $11.5 \mathrm{~mL}$ of $\mathrm{CH}_{3} \mathrm{I}$, all while stirring rapidly with a magnetic stirrer. Using $200.0 \mathrm{~mL}$ ethanol, the product was precipitated, centrifuged, washed twice with acetone over a sintered glass filter, and dried at decreased pressure using a vacuum pump. The resulting product was dissolved in $80.0 \mathrm{~mL}$ of NMP and heated to $60{ }^{\circ} \mathrm{C}$ once more. Then, with fast stirring, $4.8 \mathrm{~g}$ of $\mathrm{NaI}, 11.0 \mathrm{~mL}$ of $\mathrm{NaOH}$ aqueous solution $\left(15 \%\right.$, w/v), and $7.0 \mathrm{~mL}$ of $\mathrm{CH}_{3} \mathrm{I}$ were added, and the mixture was heated for another $30 \mathrm{~min}$. Stirring was maintained for another $1 \mathrm{~h}$ after adding $2.0 \mathrm{~mL}$ of $\mathrm{CH}_{3} \mathrm{I}$ and $0.6 \mathrm{~g}$ pellets of $\mathrm{NaOH}$. The resulting mixture was centrifuged, washed twice with acetone on a sintered glass filter, and dried under reduced pressure after being precipitated with $200.0 \mathrm{~mL}$ ethanol. To exchange the iodide ions $\left(\mathrm{I}^{-}\right)$with chloride ions, the resultant quaternary trimethyl chitosan iodide was dissolved in $40.0 \mathrm{~mL}$ of sodium chloride $(\mathrm{NaCl}, 10 \% \mathrm{w} / \mathrm{v})$. The resulting mixture was precipitated with $200 \mathrm{~mL}$ ethanol, centrifuged, and the

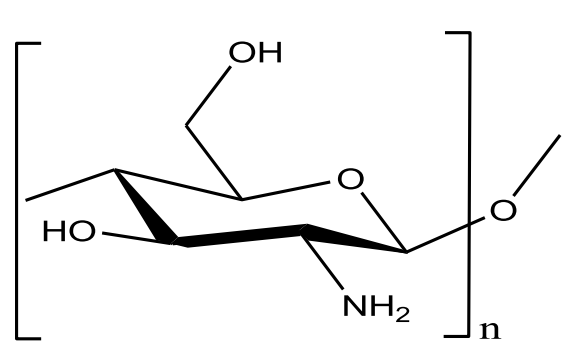

CTS
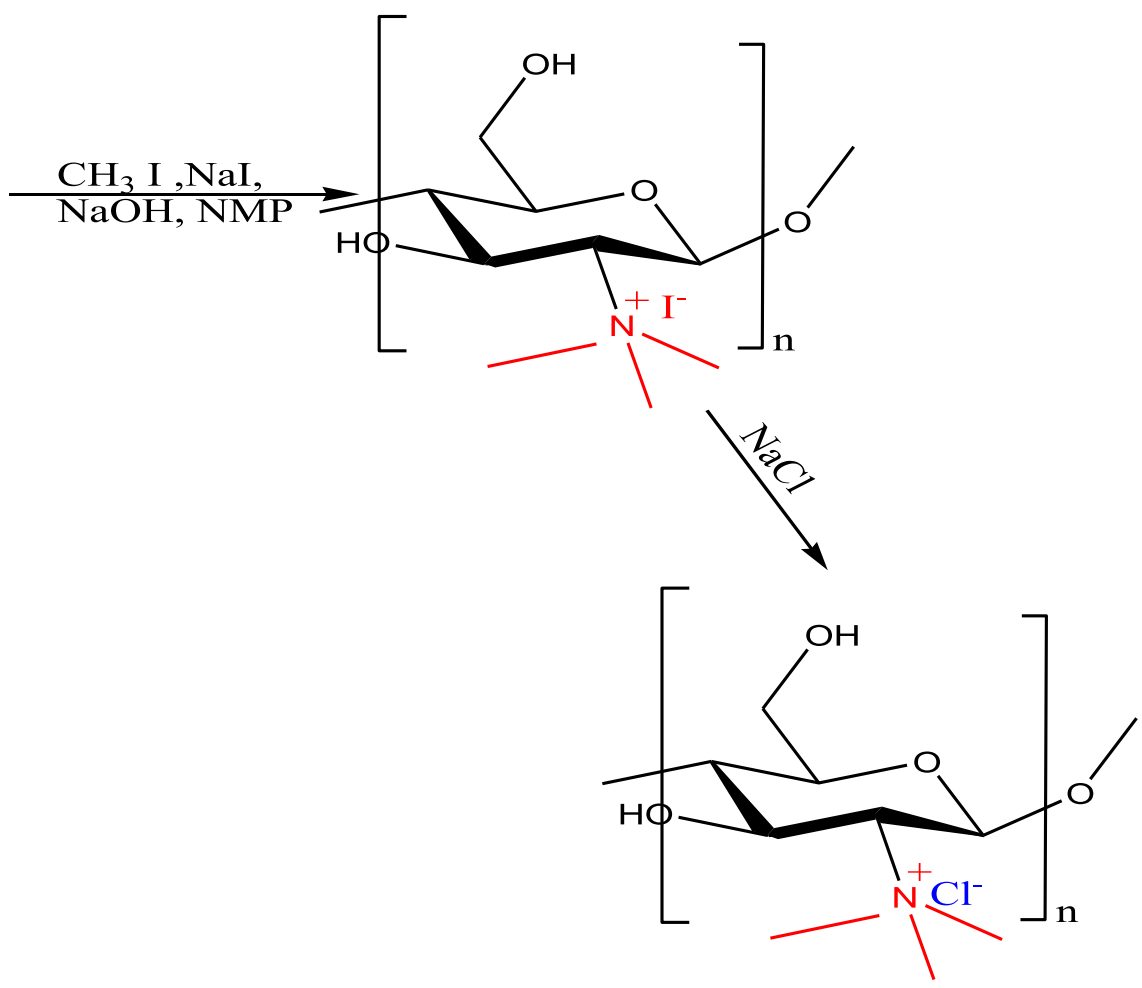

QTMC

Scheme 1 The synthetic pathway of QTMC 
supernatant containing excess $\mathrm{NaCl}$ was removed entirely. Using ethanol and ether, the result was filtered and carefully washed. The final result (a white water-soluble powder) was then vacuum-dried at $40{ }^{\circ} \mathrm{C}$.

The DQ and DT of synthesized QTMC were determined from the ${ }^{1} \mathrm{H}$ NMR data (Fig. S1) according to the equations (Eq. 1-3) [2, 5].

$\mathrm{Y}=\left[\frac{\mathrm{TML}}{9}\right]+\left[\frac{\mathrm{DML}}{6}\right]+\left[\frac{\mathrm{ACL}}{3}\right]$

$\mathrm{DQ}(\%)=\left[\frac{\mathrm{TML}}{9} \times \frac{1}{\mathrm{Y}}\right] \times 100$

$\mathrm{DT}(\%)=\left[\frac{\mathrm{DML}}{6} \times \frac{1}{\mathrm{Y}}\right] \times 100$

where Y denotes the peak area of hydrogen protons, TML/ DML denotes the integral of the trimethyl and dimethyl amino groups at 3.03 and $2.83 \mathrm{ppm}$ on the ${ }^{1} \mathrm{H}-\mathrm{NMR}$ spectrum of QTMC, respectively, and ACL denotes the integral of the acetyl group at $2.02 \mathrm{ppm}$ on the QTMC ${ }^{1} \mathrm{H}$ NMR spectrum.

\section{Aqueous Solubility of CTS and QTMC}

The aqueous solubility of CTS and QTMC at room temperature (RT) was investigated using the method described by Pardeshi and Belgamwar [5]. In brief, $100 \mathrm{mg}$ of CTS or QTMC sample was suspended in $10 \mathrm{~mL}$ of double distilled water and agitated at $25^{\circ} \mathrm{C}$ for $6 \mathrm{~h}$ to produce a saturated solution, after which the insoluble polymer was recovered by gravity filtering, washed with acetone, and dried overnight at $40{ }^{\circ} \mathrm{C}$ under vacuum.

\section{Synthesis of QTMC-BiNPs}

The previously reported procedure [11] was modified for the synthesize of QTMC-BiNPs as illustrated in Scheme 2. In the modified method, $0.513 \mathrm{~g}$ of ammonium bismuth citrate was dissolved in $30 \mathrm{~mL}$ of distilled water, and $4 \mathrm{~mL}$ of soluble QTMC solution of varying concentrations was added to the aforementioned solution with magnetic stirring at $25{ }^{\circ} \mathrm{C}$. After $20 \mathrm{~min}$, a droplet of $\mathrm{NaBH}_{4}$ aqueous solution $(10 \mathrm{~mL}$ of $1.0 \mathrm{M})$ was added, and the colorless solution quickly became black. After the $\mathrm{NaBH}_{4}$ aqueous solution was fully dripped off, the combination was left to react for another $2 \mathrm{~h}$. Centrifugation was used to purify the nanoparticles, which were then washed with double distilled water and ethanol. The resultant black powder was further analyzed using UV-visible spectrophotometer and other characterization techniques after being baked in a vacuum oven for $24 \mathrm{~h}$. The effect of initial concentration of QTMC was also monitored by varying the saccharide unit of QTMC.

\section{Preparation of Standardized Inoculum and 96 Well-Plates for Testing QTMC-BiNPs}

The MIC of the QTMC-BiNPs was determined by resazurin microtitre assay (microdilution method) [22]. E. coli and S. aureus were grown overnight in muller Hinton broth, with the final turbidity set to 0.5 McFarland Standard. The synthesized QTMC-BiNPs were introduced to muller Hinton broth in serial two-fold dilutions from 1000 to $0.98 \mathrm{~g} / \mathrm{mL}$ for the MIC test. Each microtiter plate had growth and sterility control wells, and the plates were incubated at $37{ }^{\circ} \mathrm{C}$ for $24 \mathrm{~h}$ in a bacteriological incubator. The greatest concentration of QTMC-BiNPs was found in row 1 of the microtiter plate. Row 12 was used as a control (medium and bacterial inoculums). Following the incubation period, each well of the microtiter plate was filled with $30 \mathrm{~mL}(0.015 \%)$ resazurin solution and incubated at $37{ }^{\circ} \mathrm{C}$ for 2 to $4 \mathrm{~h}$ to observe color change [20, 23, 24]. MBC was also calculated by collecting the contents of wells in three plates with concentrations greater than the MIC value. When there was no colony development in the immediately plated wells, the MBC value was carefully evaluated. In

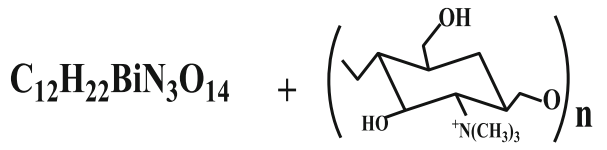

Ammonium bismuth citrate QTMC

Scheme 2 Synthetic pathway for QTMC-BiNPs

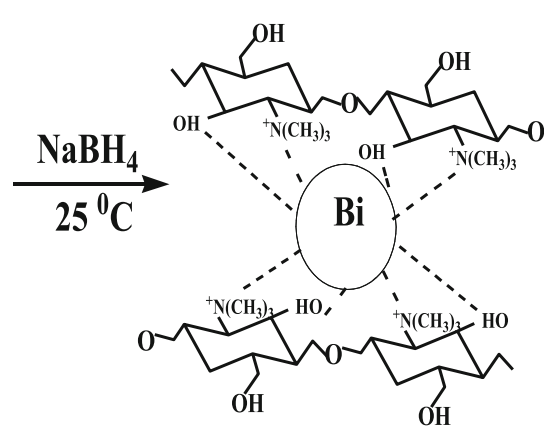

QTMC-BiNPs 
addition, the contents of the wells that showed signs of growth inhibition were serially diluted to determine the bacteria's end-point death [22].

\section{Catalysis}

\section{Assessment of QTMC-BiNPs Catalytic Activity}

Following a previously described approach [11], the catalytic activity of QTMC-BiNPs in the reduction of 4-NP by $\mathrm{NaBH}_{4}$ was studied. A freshly prepared aqueous solution of $\mathrm{NaBH}_{4}(0.4 \mathrm{~mL}, 33.4 \mathrm{~g} / \mathrm{L})$ was added to a $2.4 \mathrm{~mL}$ 4-NP aqueous solution $(0.21 \mathrm{~g} / \mathrm{L})$ with continual magnetic stirring at room temperature for the catalytic reduction of 4-NP. Following the addition, the color of the solution changed from pale yellow to deep yellow. The reaction mixture was then added to the QTMC-BiNPs suspension in water $(0.4 \mathrm{~mL}, 2 \mathrm{~g} / \mathrm{L})$. The deep yellow vanished almost instantly when the catalyst was added, and the mixture turned colorless. The concentration of the QTMC-BiNPs solution in each well was monitored using ICP-OES. The ratio of the absorbance at $\mathrm{t}=\mathrm{t}\left(\mathrm{A}_{\mathrm{t}}\right)$ to that at $\mathrm{t}=0\left(\mathrm{~A}_{0}\right)$ is equal to the concentration ratio $C_{t} / C_{0}$ of 4-NP since its UV absorbance is directly proportional to its concentration in the solution. As a result, the absorption intensity was used to directly monitor the conversion process. At room temperature, a UV-visible spectrophotometer was used to track the progress of the conversion of 4-NP to 4-AP.

\section{Evaluation of Degree of Reusability of QTMC-BiNPs}

QTMC-BiNPs were recycled by centrifugation to test the catalyst's reusability. The catalyst was rinsed with ethanol, diethyl ether, and distilled water after separation from the reaction solution. The re-activated catalyst was then dried and utilized repeatedly eight times (8) for the reduction reaction.

\section{Characterization}

${ }^{1} \mathrm{H}$ NMR spectrum of QTMC was determined on Bruker Advance III $400 \mathrm{MHz}$ by measuring approximately $10 \mathrm{mg}$ of QTMC in a clean NMR tube and dissolved in $\sim 0.5$ $\mathrm{mL}$ of deuterated water $\left(\mathrm{D}_{2} \mathrm{O}\right)$. The UV-Visible spectra of CTS, QTMC and QTMC-BiNPs were all recorded on UVVisible Spectrometer (Thermo Scientific Evolution 201 and Shimadzu UV-2600 (solid sample). The UV-Vis spectra were obtained in the $200-800 \mathrm{~nm}$ region. CTS, QTMC, and QTMC-BiNPs FTIR spectra were acquired using a single ATR-FTIR spectrometer (PerkinElmer Spectrum Two) with a scan range of $4000-400 \mathrm{~cm}^{-1}$ and a resolution of $4 \mathrm{~cm}^{-1}$. Thermogravimetric (TGA/DTG) analysis of CTS, QTMC, and QTMC-BiNPs was carried out using a Thermogravimetric Analyzer (SDT Q600 V20.9 Build 20, Universal V4.5A TA Instrument, IUIC, Mahatma Gandhi University, India) at a heating rate of $10{ }^{\circ} \mathrm{C}$ min 1 from 40 to $700{ }^{\circ} \mathrm{C}$, with a nitrogen purge of $50 \mathrm{~mL} / \mathrm{min}$. The X-ray diffractograms of QTMC-BiNPs, was recorded on an X-ray diffractometer (Brucker AXS D8 advance ${ }^{\circledR}$, Karlsruhe, Germany). To separately evaluate the concentration, an Inductively Coupled Plasma Optical Emission Spectrometer (ICP-OES 3000, Technologies Analytical Company, India) was used. Diffractograms were scanned with a resolution of $0.02^{\circ}$ and a scanning speed of $2.0^{\circ} \mathrm{min}^{-1}$ in a scattering range of $20^{\circ}-80^{\circ}(2)$, using an accelerating voltage of $40 \mathrm{kV}$ and a current intensity of $35 \mathrm{~mA}$. The dispersions of QTMC-BiNPs were seen using a high-resolution transmission electron microscope (HRTEM, JEOL, JEM-2100, MA, Japan). Carbon-coated copper grids were dyed with phosphotungstic acid $(2 \% \mathrm{w} / \mathrm{v})$, air-dried, and examined under HR-TEM. Photomicrographs were taken at $200 \mathrm{kV}$ at various magnifications. The morphology of CTS, QTMC, and QTMC-BiNPs was examined using scanning electron microscopy (SEM, JSM$6390 \AA$, Jeol DATUM Ltd., Tokyo, Japan). A thin coating (400 A) of gold was sputtered-coated onto the samples. Photomicrographs were taken at a voltage of $10 \mathrm{kV}$ and a magnification of 500 above.

\section{Results and Discussion}

\section{Characterization of CTS, QTMC and QTMC-BiNPs}

The ${ }^{1} \mathrm{H}$ NMR spectrum of QTMC obtained after two step methylation of CTS is presented in Fig. S1. The spectrum clearly revealed peaks at 3.03 and $2.83 \mathrm{ppm}$ assigned to $\mathrm{H}$ of $\mathrm{N}$-trimethyl groups and $\mathrm{H}$ of $\mathrm{N}$-dimethyl groups, respectively. The characteristics peaks observed between 4.6 and $5.4 \mathrm{ppm}$ can be assigned to $\mathrm{H}$ proton at $\mathrm{C} 1$ and the peak at $2.02 \mathrm{ppm}$ can be ascribed to $\mathrm{H}$ of $\mathrm{N}$-acetyl group. Comparing ${ }^{1} \mathrm{H}$ NMR spectrum of CTS of the same molecular weight and degree of deacetylation reported by Pardeshi and Belgamwar [5] with ${ }^{1} \mathrm{H}$ NMR spectrum of QTMC, the characteristic peaks attributed to N-trimethyl and $\mathrm{N}$-dimethyl groups were absent which confirmed the successful quaternization of CTS. The DQ and DT of QTMC were evaluated according to Eq. 1-3. The DQ and DT of QTMC as calculated from proton NMR spectra were $63.33 \%$ and $11.75 \%$, respectively. These findings confirm that the main product is a quaternary derivative.

The formation of soluble QTMC and the addition of hydrophilic groups to the CTS backbone resulted in a significantly increased aqueous solubility of QTMC, with percentage solubility of $93.01 \%$ (QTMC 1, first trial) and 95.75\% (QTMC 2, second trial), which was approximately 
25-folds greater than CTS (4\%) (Fig. S2). In comparison to native CTS, trimethylation of CTS by adding hydrophilic functional groups increased QTMC solubility [25]. The UV-visible spectrum of CTS displayed a prominent absorption peak at $220 \mathrm{~nm}$ (Fig. 1a). Expectedly, no absorption peaks observed in the range of $300-800 \mathrm{~nm}$. This observation agreed perfectly with previous study commonly used for the identification of CTS [26]. Also, the UV-visible spectrum of QTMC in (Fig. 1b) displayed characteristic bands at 218,290 and $360 \mathrm{~nm}$ which is completely different from the absorption spectrum of native CTS. As shown in Fig. 1c, the formation of colloidal suspensions of QTMC-BiNPs was first evident from visual inspection of the reagent mixtures under magnetic stirring. The absorption peak centered at $275 \mathrm{~nm}$ is observed as a plasmon band attributable to QTMC-BiNPs (Fig. 1c). Das shows a slight bathochromic shift compared with the reported work of Ma and co-workers [27] because of the aggregated status of their Bi nanoparticles and it is in good agreement with the theoretical spectrum of larger particles and with a similarly calculated spectrum for colloidal bismuth reported elsewhere [28]. Furthermore, the influence of QTMC starting concentration on the formation of QTMC-BiNPs was investigated, and absorption intensity reduced as QTMC saccharide unit concentration increases (Fig. 1d).

The overlapped ATR-FTIR spectral of CTS, QTMC and QTMC-BiNPs are presented in Fig. 2. The presence of a vibrational frequency at $1476 \mathrm{~cm}^{-1}$ on the QTMC spectrum attributed to angular distortion of $\mathrm{C}-\mathrm{H}$ bonds of methyl groups, which is completely absent on the spectrum of ordinary CTS, suggests that CTS has been quaternized.
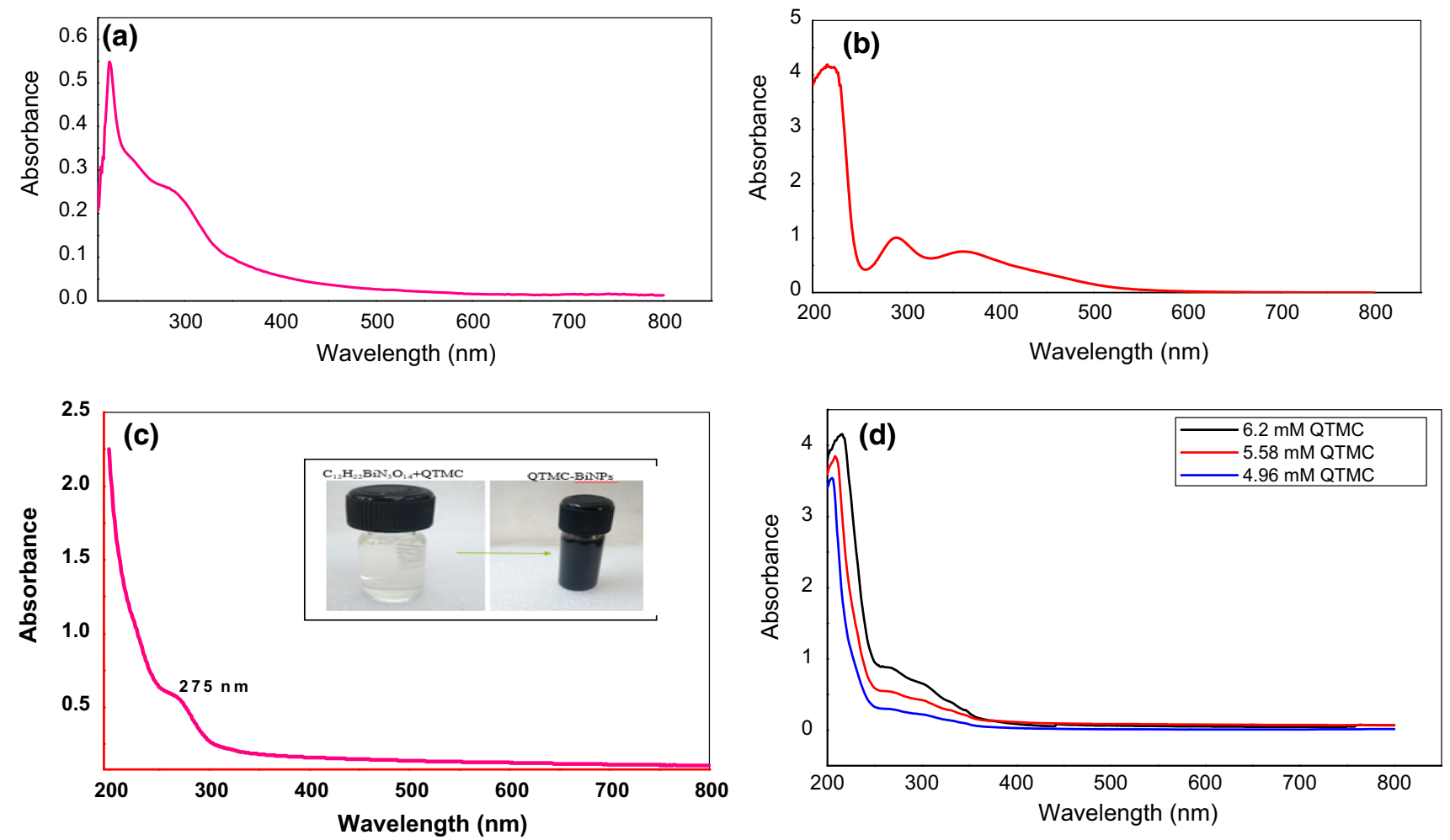

Fig. 1 UV-Visible spectral of a CTS b QTMC c QTMC-BiNPs d QTMC-BiNPs at varied concentration of QTMC

and coworkers recently revealed that BiNPs had a distinctive absorption peak at $270 \mathrm{~nm}$, which is almost identical to the absorption band found in our study [24]. Similarly, a prior study found a UV-Vis absorption peak at $285 \mathrm{~nm}$ in nanometer-sized bismuth particles [27]. However, the usual plasmon band of BiNPs is between 250 and $290 \mathrm{~nm}$, implying that the BiNPs formed in this study. The absorption peak at $275 \mathrm{~nm}$ of QTMC-BiNPs in this study
Another indicator of trimethylation is hypsochromic shift in the vibrational frequency of $2876 \mathrm{~cm}^{-1}$ on the CTS spectrum to $2935 \mathrm{~cm}^{-1}$ on the QTMC spectrum (axial distortion of $\mathrm{C}-\mathrm{H}$ bonds). When compared to the CTS spectrum, the large amount of $\mathrm{C}-\mathrm{H}$ bonds of methyl groups on QTMC may be responsible for pushing such band to a higher wavenumber on the QTMC spectrum. Primary and secondary alcohol vibrational frequencies at 1151 and 1027 


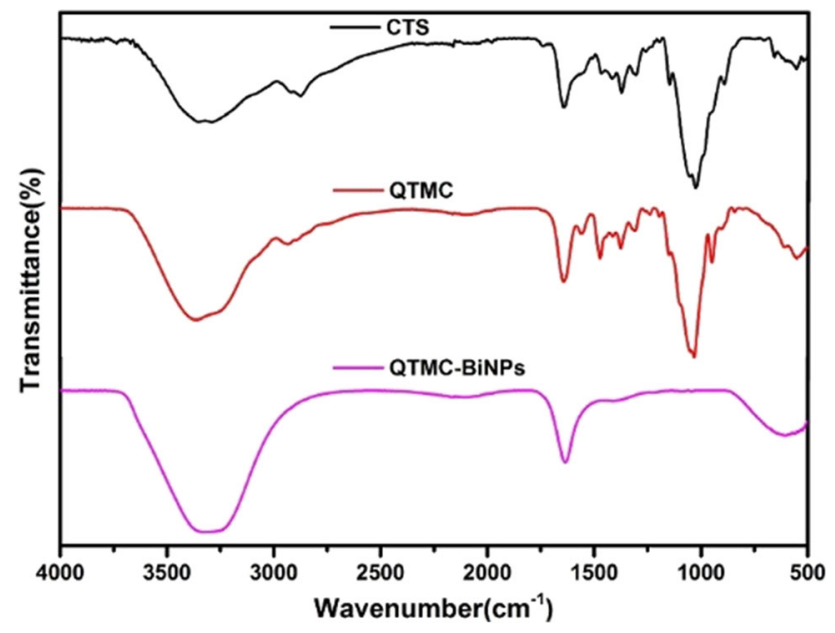

Fig. 2 ATR-FTIR spectra of CTS, QTMC and QTMC-BiNPs

$\mathrm{cm} 1$ on the CTS spectrum remain constant at 1151 and $1032 \mathrm{~cm} 1$ on the QTMC spectrum. The conclusion that can be made from this is that only amino groups and neither C-3 or C- 6 on the CTS are involved in the substitution process $[2,5]$.

In the ATR-FTIR spectrum of QTMC-BiNPs, the disappearance of bands at 1380,1476 , and $2935 \mathrm{~cm}^{-1}$ indicates the partial loss of the $\mathrm{C}-\mathrm{H}$ vibrations assigned to the methyl groups changed on the $\mathrm{C}-2$ position of native CTS (Fig. 2). BiNPs and QTMC biopolymeric ligand interaction is suggested by the reduction and broadening of absorption peaks in the $600-2000 \mathrm{~cm}^{-1}$ range. The aforementioned finding is consistent with a previous research of Ding and colleagues [29], who found that the interaction of gold metal with chitosan derivative reduced the capping agent's peak intensity.

The phase and crystallinity of the synthesized QTMCBiNPs was investigated by X-ray diffraction (Fig. 3). According to the crystallographic spectrum, all of the diffraction peaks can be attributed to the pure rhombohedral phase of Bi (JCPDS No. 05-0519), which is consistent with previous research $[11,30]$. The most intense peak at $2 \theta=27.23$ is obtained along (012) reflection plane and it is very close to the position of the strongest line $(2 \theta=29.32)$ of the reference bismuth [30]. This XRD pattern suggests that the final product is elemental bismuth, not bismuth oxide, given the current synthetic circumstances.

The X-ray photoelectron spectroscopy (XPS) spectrum of QTMC-BiNPs is presented in Fig. 4a. The spectrum revealed elements such as bismuth $(\mathbf{B i} \mathbf{4 f})$, nitrogen $(\mathbf{N} \mathbf{1 s})$, chlorine (Cl 2p), oxygen (O 1s) and carbon (C 1s). Figure $4 \mathrm{~b}$ displayed the spectrum of $\mathrm{Bi} 4 \mathrm{f}$ core level of QTMC-BiNPs with corresponding binding energies. The two diffraction peaks at $163 \mathrm{eV}$ and $157.1 \mathrm{eV}$ can be

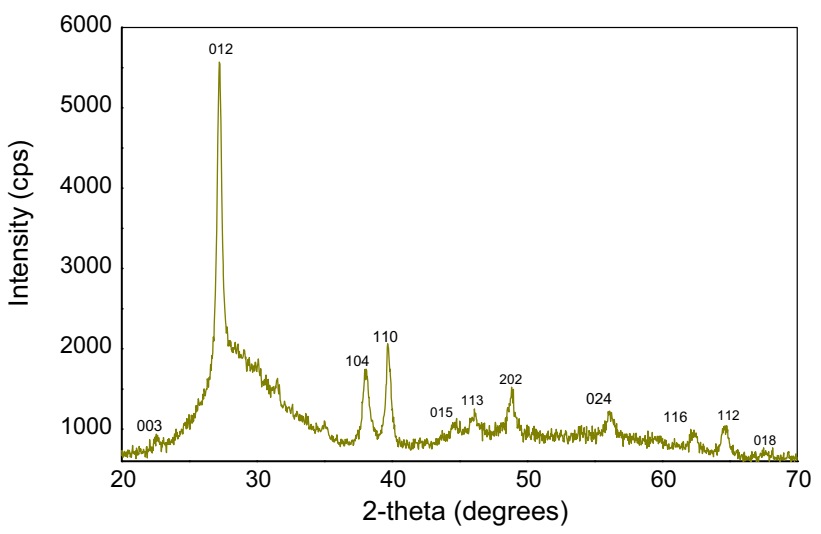

Fig. 3 XRD diffraction patterns of QTMC-BiNPs

attributed to $\mathbf{B i} \mathbf{4} \mathbf{f}_{5 / 2}$ and $\mathbf{B i} \mathbf{4} \mathbf{f}_{7 / 2}$, respectively revealing the purity of the synthesized QTMC-BiNP [31]. It is worth noting that the XPS spectrum of $\mathrm{Bi} 4 \mathrm{f}$ region revealed no shoulder binding energies. This implies that there are no traces of bismuth oxide nanoparticles in the QTMC-BiNPs colloidal formation.

The SEM micrograph of CTS (Fig. S3a) displayed at image focus zoom of $10 \mu \mathrm{m}$ indicated smooth surface with
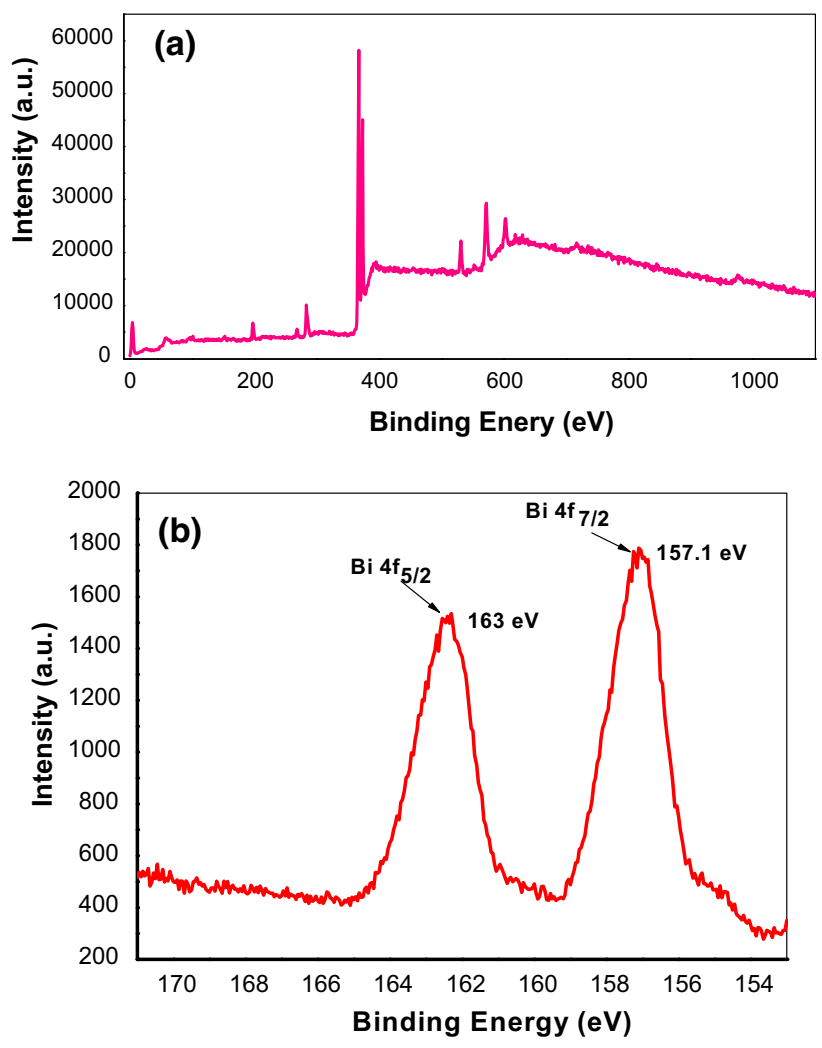

Fig. 4 XPS survey of a QTMC-BiNPs b Bi 4 f core levels of QTMCBiNPs 
some straps indicating the degree of chitin deacetylation. This morphological characteristic is in agreement with the previous studies $[2,5,26]$. In contrast to the micrograph of CTS, the SEM micrograph of QTMC at image focus zoom of $5 \mu \mathrm{m}$ as depicted in Fig. S3b revealed a uniformly distributed cubic like morphology. This significant morphological characteristic confirmed the successful methylation of CTS with high degree of methylation. SEM micrographs of QTMC-BiNPs at image focus zooms of 10 and $50 \mu \mathrm{m}$ (Fig. S2d) displayed a quasi-spherical shape with some traces of rod like morphology.

The HR-TEM images of QTMC-BiNPs as shown in Fig. $5 \mathrm{a}$ and $\mathrm{b}$ at image focus zoom of 50 and $20 \mathrm{~nm}$ were quasi-spherical in shape with some straps of rod like morphology and the images clearly displayed well capped nanostructures. The average particle size is between the range of $30-45 \mathrm{~nm}$ in diameter as estimated by image J software confirming the nano dimension of the bismuth metal. Its selected area of electron diffraction (SAED) patterns shows the distinct patterns of rhombohedral metallic Bi nanoparticles, as determined by d-spacing (JCPDS File Card no: 05-0519). Figure 5c shows the distinctive lattice fringes and d-spacing calculated using Gatan Digital Micrography software, which is $0.32 \mathrm{~nm}$ and corresponds to the (012) hkl planes of the rhombohedral bismuth crystal (JCPDS File with Card No: 05-0519 [32]. The SAED patterns revealed the (202), (113), (112), (110), (104), (101), (024), (018), (012), and (003) hkl crystalline planes of rhombohedral bismuth (Fig. 5d).

The EDX spectrum of QTMC-BiNPs in Fig. S4 indicated the presence of Bi metal in QTMC-BiNPs suggesting that QTMC-BiNPs were successfully synthesized. The carbon $(\mathrm{C})$ and oxygen $(\mathrm{O})$ peaks may be generated from the QTMC while the copper $(\mathrm{Cu})$ observed was due to the grid of $\mathrm{Cu}$ used in sample preparation for TEM analysis.

CTS and QTMC TGA profiles are shown in Figs. S5 and S6, respectively. The evaporation of water in the biopolymers caused the initial thermal event for CTS and QTMC, which was detected in the range of $50-150{ }^{\circ} \mathrm{C}$ with weight loss of $1.815 \%$. The thermal event indicated that CTS had a higher water content than QTMC. Related to the inclusion of positive charge on the nitrogen atoms, QTMC is more cationic and hence has a higher solubility in water than CTS, which is due to the methylation of nitrogen atoms. The QTMC, on the other hand, has a large concentration of methyl groups, which might explain its hydrophobic behavior. This property is influenced by QTMC's DQ and molecular weight [11]. Furthermore, CTS contains
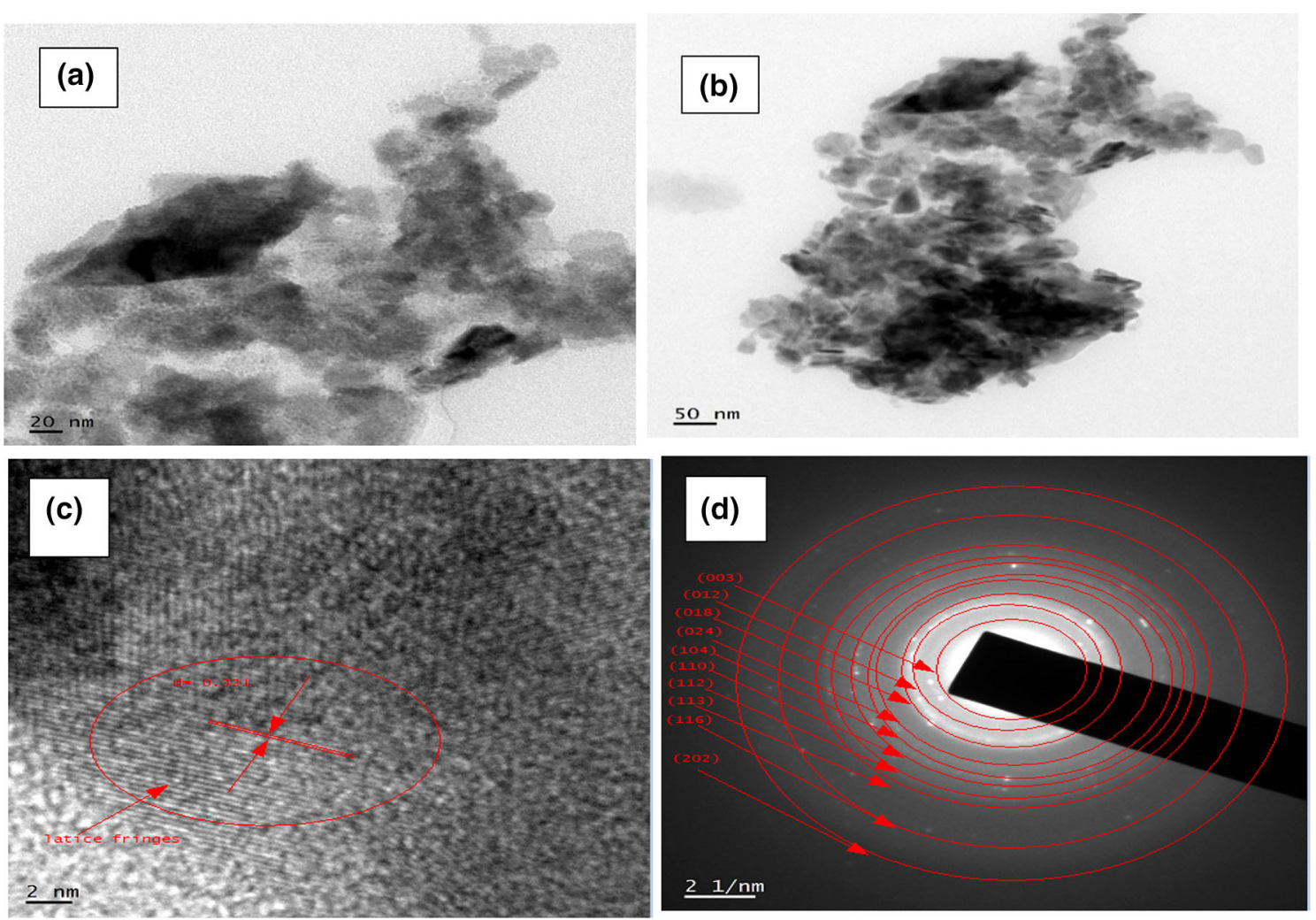

Fig. 5 a HR-TEM image of QTMC-BiNPs at image focus zoom of $50 \mathrm{~nm}$ b HR-TEM image of QTMC-BiNPs at image focus zoom of $20 \mathrm{~nm}$ zoom $\mathbf{c}$ d- spacing and lattice fringes of QTMC-BiNPs d HR-TEM selected area of diffraction patterns of indexed QTMC-BiNPs 
functional groups like hydroxyl $\left(\mathrm{OH}^{-}\right)$and amino $\left(\mathrm{NH}_{2}\right)$ that is capable of providing dipole-dipole or ion-dipole interactions with water. The high DQ and molecular weight of QTMC should allow it to interact with water more effectively. These interactions occurred to a lower extent in QTMC, which might be owing to its hydrophobic nature [3]. This explains why CTS has more water than QTMC. The second thermal decomposition observed in the range of $225-300{ }^{\circ} \mathrm{C}$ for CTS and $200-250{ }^{\circ} \mathrm{C}$ for QTMC can be attributed to the degradation of biopolymers (CTS and QTMC).

The DTG curves confirmed that the CTS at $300.09{ }^{\circ} \mathrm{C}$ (Fig. S5) is more thermally stable than QTMC at $223.43{ }^{\circ} \mathrm{C}$ (Fig. S6). The high DQ (63.33\%) could responsible for the less thermal stability of QTMC. This finding is in line with the reported work of Pardeshi and Belgamwar [5], where the study observed $297.91{ }^{\circ} \mathrm{C}$ for chitosan and $221.76{ }^{\circ} \mathrm{C}$ for trimethyl chitosan. The high number of methyl groups inserted on nitrogen atoms may have lowered intra-chain strength; particularly those from H-bonds, this might be another explanation for QTMC's lower thermal stability when compared to CTS. Another factor that may have contributed to QTMC's low water content is its exposure to dryness during synthesis.

The TGA and DTG thermal profiles of QTMC-BiNPs are presented in Fig. S7 showed the TGA and DTG thermographs of QTMC-BiNPs. According to the TGA/DTG curve, there are two stages of degradation in QTMC-
BiNPs. The evaporation of physically held liquid caused the first thermal event, which occurred between 25 and $100{ }^{\circ} \mathrm{C}$ and resulted in a $14.17 \%$ weight loss $(2.305 \mathrm{mg})$. The decomposition of the QTMC polymeric ligand which is the second thermal event, took place between the range of $225-300{ }^{\circ} \mathrm{C}$ with a $20.66 \%$ weight loss $(3.362 \mathrm{mg})$.

\section{Antibacterial Activities}

The antibacterial activity of QTMC-BiNPs was tested against $E$. coli and $S$. aureus. In the presence of QTMCBiNPs, the estimated MIC and MBC were determined. Figure 6 shows the resazurin microtiter test plates for the synthesized QTMC-BiNPs. The calculated MIC values against E. coli and $S$. aureus in the presence of QTMCBiNPs were $15.63 \mathrm{~g} / \mathrm{mL}$ and $125 \mathrm{~g} / \mathrm{mL}$, respectively while the MBC of 31.25 and $500.00 \mu \mathrm{g} / \mathrm{mL}$ were estimated against E. coli and S. aureus, respectively. The study painstakingly compared the MIC value obtained with some published research works on antibacterial activity of $\mathrm{Bi}$ nanoparticles. Das and colleagues [24] reported in their recently published experimental work on antibacterial activity of BiNPs that the MIC value recorded against $S$. aureus and E. coli were $250 \mu \mathrm{g} / \mathrm{mL}$ and $500 \mu \mathrm{g} / \mathrm{mL}$ respectively. Similarly, Rieznichenko et al. also examined the antibacterial activity of $40 \mathrm{~nm}$ BiNPs recently and a terminal concentration of $12.6 \mathrm{mg} / \mathrm{mL}$ was reported for E. Coli pathogen [20]. In a related work, Hernadez-
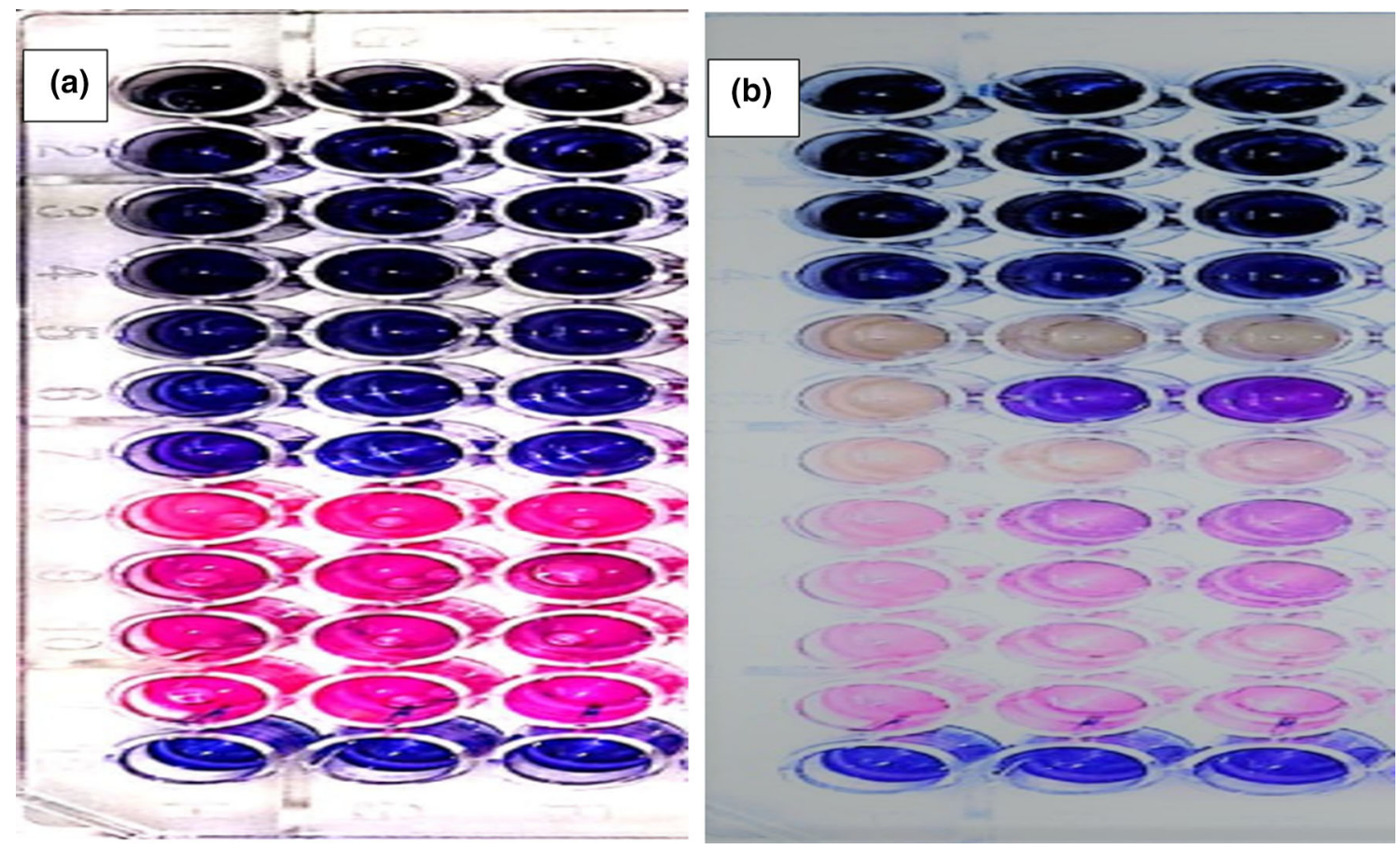

Fig. 6 Resazurin microtiter assay plates for the antibacterial activity of QTMC-BiNPs against a E. coli $\mathbf{b}$ S. aureus 
Delgadillo and co-workers indicated that Bi nanoparticles can inhibit bacteria at minimum concentration of $0.5 \mathrm{mM}$ [23]. Based on the compared results, it is crystal clear that the antibacterial activities of QTMC-BiNPs in this present study is very promising and it stands a better chance to fight infectious disease but more testing is needed to ensure its safety in human.

Apparently, the mechanisms of action of metal nanoparticles are difficult to predict and still a subject of extensive scientific research. However, this study proposed bimodal mechanism of action from the literature [33] as illustrated in Fig. 7. The following could possibly lead to the death of cells of bacteria used in this study; disruption of cell wall synthesis (A), penetration of QTMC-BiNPs into the periplasmic space (B), cell pits caused by QTMCBiNPs (C), improper function of ribosome (D), reactive oxygen species (ROS) production (E), interaction of QTMC-BiNPs with protein, cystine to be specific (F) and inhibition DNA replication by QTMC-BiNPs.

\section{Catalysis Result}

\section{QTMC-BiNPs' Catalytic Activity}

In the presence of $\mathrm{NaBH}_{4}$, the reduction of 4-NP over metal nanoparticles, particularly noble metal nanoparticles, is an efficient strategy for producing 4-AP [4, 21]. Despite several studies on noble metal nanoparticles, there have been few publications on the catalytic reduction of 4-NP by inexpensive and easily available bismuth nanoparticles [21]. Figure 8 depicts the reaction pathway of 4-NP conversion. The conversion of 4-NP to 4-AP using aqueous
$\mathrm{NaBH}_{4}$ solution appears to be thermodynamically advantageous. However, the reaction's viability is reduced due to a kinetic barrier created by a high potential difference between the donor and acceptor molecules [11]. As a result, during the reduction process, the metal initiates catalytic reduction by relaying electrons from the donor $\mathrm{BH}_{4}{ }^{-}$to the acceptor 4-NP that is adsorbed on the catalysts [34]. Scheme 3 depicts a schematic representation of the proposed catalytic reduction.

The catalytic activity of QTMC-BiNPs was investigated using this process. The absorption spectra of 4-NP before reaction exhibited a strong absorption peak at $316 \mathrm{~nm}$ and a minor peak at $400 \mathrm{~nm}$ (Fig. 8b). The solution transformed from light yellow to brilliant yellow with the addition of $\mathrm{NaBH}_{4}$, and the distinctive absorption peak experiences a bathochromic shift from 316 to $400 \mathrm{~nm}$, suggesting the establishment of longer conjugative bonds with the nitrophenolate ion [21, 35, 36]. Eventually, after the addition of QTMC-BiNPs, the nitrophenolate ions peak at $400 \mathrm{~nm}$ decreases tremendously to almost zero absorbance and only a peak at $300 \mathrm{~nm}$ can be observed which interpret to the disappearance of 4-NP and appearance of 4-AP [11, 21, 37].

The absence of absorption at $400 \mathrm{~nm}$ indicates that the reactants have been completely converted to product. When 4-NP was reduced to 4-AP without a catalyst, the reaction time was $430 \mathrm{~min}$, but with a catalyst, the reaction time was finished in $160 \mathrm{~s}$. Figure $8 \mathrm{c}$ depicts the time-dependent decrease of 4-NP spectra in the presence of QTMC-BiNPs. As the reaction continues, it is evident that the distinctive absorption band of 4-NP declines while the absorption spectrum of 4-AP rises. It is worth noting that

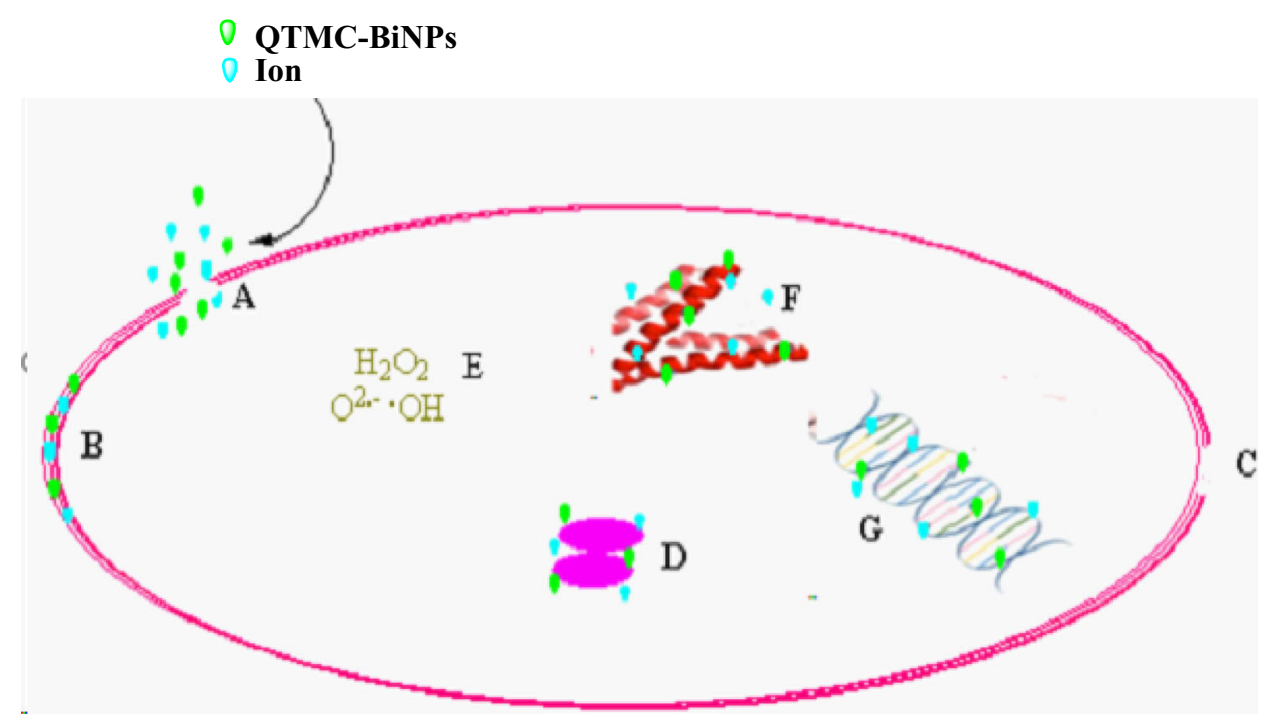

Fig. 7 Proposed multi target mechanism of action of QTMC-BiNPs 
(a)
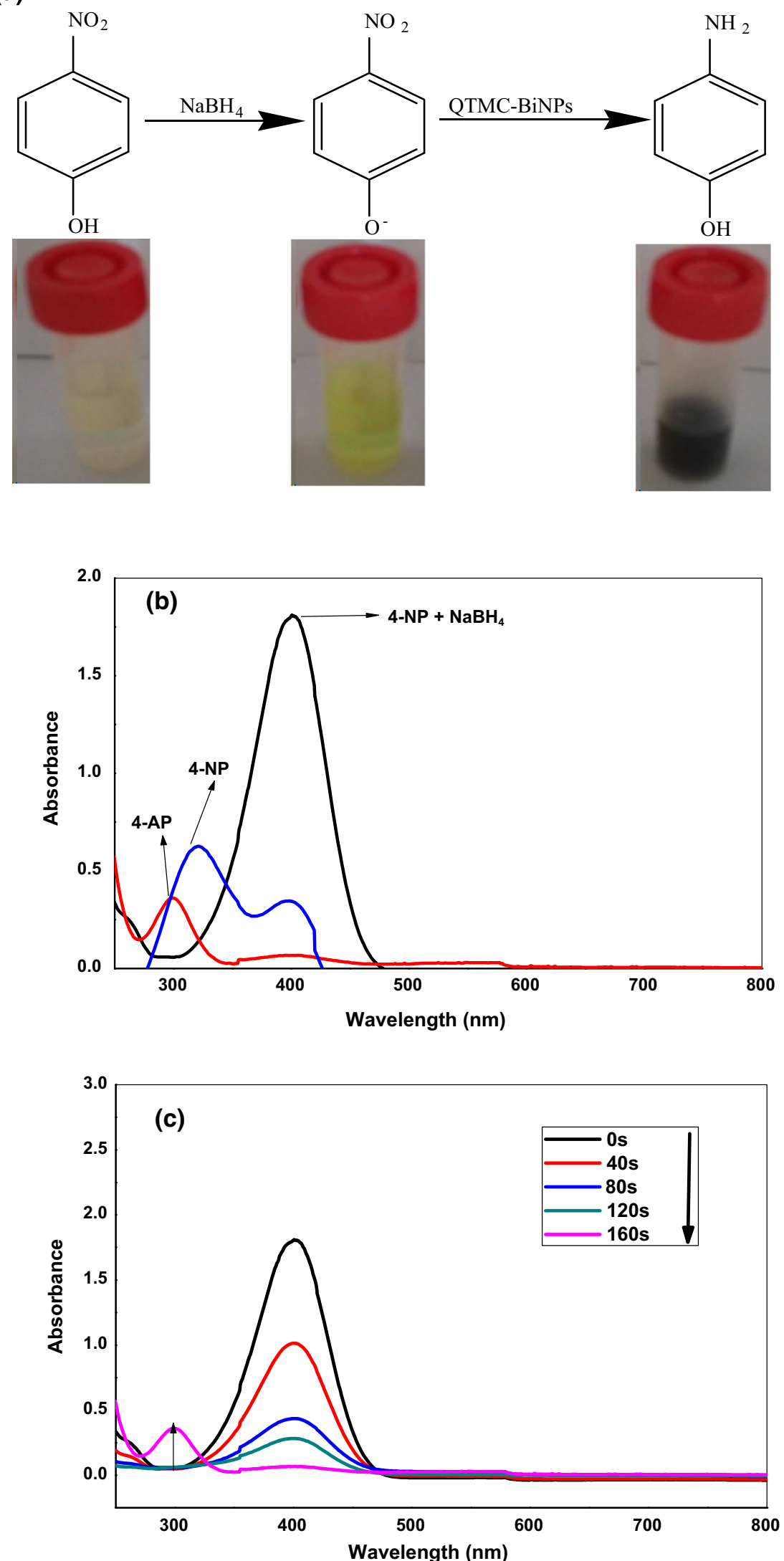

Fig. 8 a Conversion of 4-nitrophenol to 4-aminophenol and colour change observed, UV-Vis absorption spectral of $\mathbf{b}$ 4-nitrophenol before and after reduction catalyzed by QTMC-BiNPs c Time-dependent reduction of 4-nitrophenol to 4-aminophenol in the presence of QTMC-BiNPs 
the rise in the absorption band of 4-AP was insignificant from 0 to $120 \mathrm{~s}$, but it increased dramatically at $160 \mathrm{~s}$ when the 4-NP completely vanished. The time-dependent absorption spectra made it simple to monitor the reaction

\section{Extent of Reusability of QTMC-BiNPs}

Reusability and stability are critical variables to consider when selecting a catalyst. The degree of the catalyst's

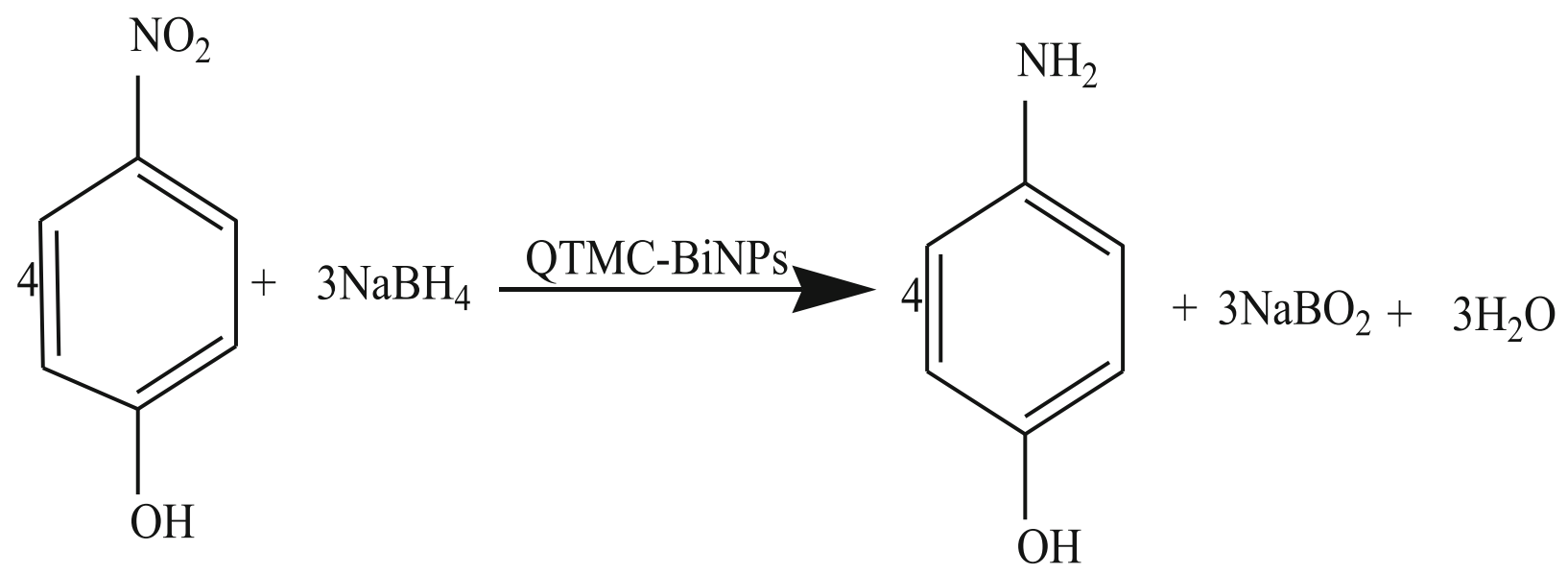

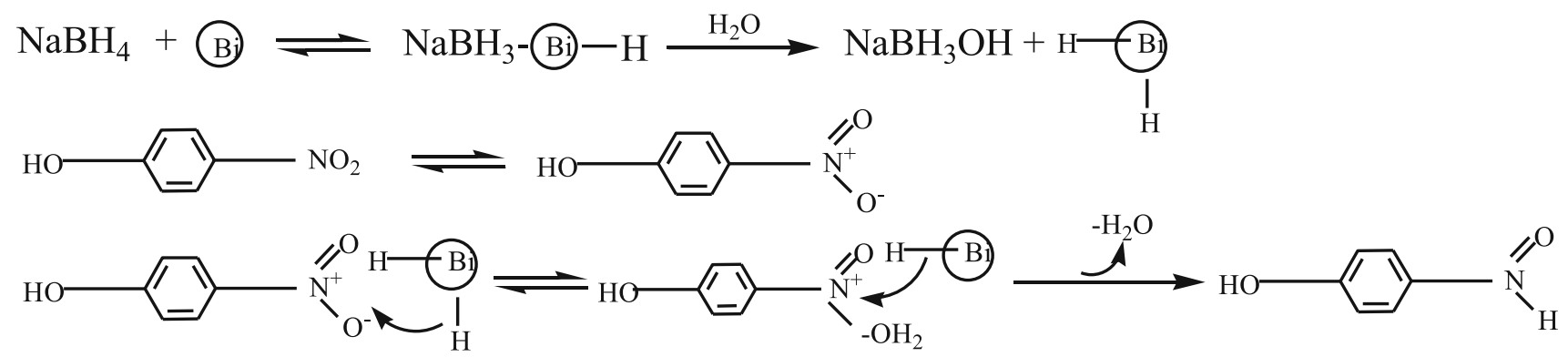

Where (B) $=$ QTMCBiNPs

Scheme 3 Mechanism of 4-NP reduction catalyzed by QTMC-BiNPs

kinetics. Because the concentration of $\mathrm{NaBH}_{4}$ was higher than that of 4-NP, the reaction kinetics were considered to be pseudo-first order [38]. Thus, the pseudo-first order rate constant of the reaction was derived using the equation $\ln$ $\left(A_{t} / A_{0}\right)=K_{t}$, where $A_{0}$ and $A_{t}$ were the starting and time $t$ absorbance values of 4-NP, respectively. The rate constant calculated from the slope of the plot was $0.02542 \mathrm{~s}^{-1}$. The activity factor was determined as the ratio of the rate constant $\mathrm{K}$ to the total weight of the catalyst using $\mathrm{k}=\mathrm{K} /$ $\mathrm{m}$. The $\mathrm{k}$ of QTMC-BiNPs was determined to be $\mathrm{k}=0.02542 \mathrm{~s} / 1 \mathrm{mg}=25.42 \mathrm{~s}^{-1} \mathrm{~g}^{-1}$, which is extremely similar to the activity factor previously reported for bismuth nanoparticles [11]. This study indicates that QTMCBiNPs have superior catalytic performance when compared to noble metal catalysts. reusability was depicted in Fig. 9. The same catalyst was used for the reduction process eight (8) times under the same conditions. When the catalyst was first used, the reaction time was faster than when it was used again. The response time of QTMC-BiNPs increased with the number of recycling times. The reduction in catalytic activity might be due to QTMC-BiNP aggregation and reaction product adsorption on QTMC-BiNPs.

\section{Conclusions}

A simple two step reductive methylation of CTS was used to prepare QTMC which showed excellent solubility in neutral and basic solutions and was effective in the 
stabilizing of QTMC-BiNPs. It was observed that the QTMC-BiNPs with positively charged surfaces inhibited/ killed.

E. coli and S. aureus at lower concentration compared to previously reported literatures. Furthermore, the synthesized QTMC-BiNPs showed effective catalytic abilities in

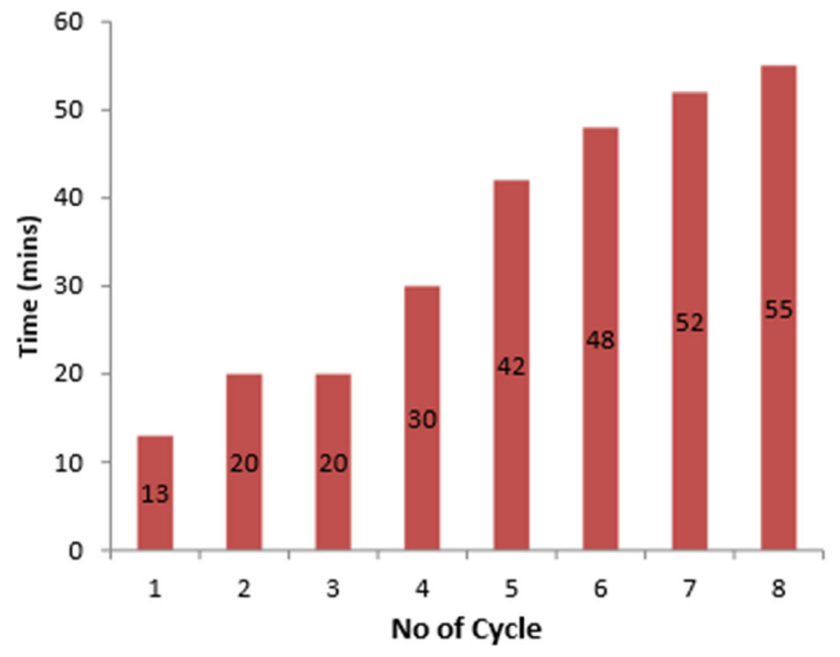

Fig. 9 Chart showing the extent of reusability of QTMC-BiNPs

the reduction of 4-NP to 4-AP. The reaction time of $160 \mathrm{~s}$ which was lower than the one reported in the literature at 42 min for chitosan- stabilized Palladium nanoparticles to catalyze the same reaction clearly showed the improvement in QTMC-stabilized nanoparticles. The nanoparticles also showed first-rate reusability as it was utilized eight times to catalyze the same reaction using the same conditions.

Supplementary Information The online version contains supplementary material available at https://doi.org/10.1007/s10876021-02156-8.

Acknowledgements Yakubu Adekunle Alli is grateful to Peculiar Ultimate Concerns Limited for funding his study travel to India. We would also like to thank the International and Inter-University Centre for Nanoscience and Nanotechnology (IIUCNN), Mahatma Gandhi University, Kottayam, Kerala, India, for providing advanced characterization tools for this study.

\section{Declarations}

Conflict of interest The authors declare that they have no competing interest.

\section{References}

1. Z. Jia, D. Shen, and W. Xu (2001). Carbohydr. Res. 333, 1-6.

2. T. C. Chang, C. C. Chen, K. M. Cheng, C. Y. Chin, Y. H. Chen, X. A. Chen, J. R. Sun, J. J. Young, and T. S. Chiueh (2017). Colloids Surf. B Biointerfaces 155, 61-70.
3. A. D. Kulkarni, H. M. Patel, S. J. Surana, Y. H. Vanjari, V. S. Belgamwar, and C. V. Pardeshi (2017). Carbohydr. Polym 157, 875-902.

4. S. K. Samal, M. Dash, S. Van Vlierberghe, D. L. Kaplan, E. Chiellini, C. van Blitterswijk, L. Moroni, and P. Dubruel (2012). Chem. Soc. Rev. 41, 7147.

5. C. V. Pardeshi and V. S. Belgamwar (2016). Int. J. Biol. Macromol 82, 933-944.

6. C. H. Kim, J. W. Choi, H. J. Chun, and K. S. Choi (1997). Polym. Bull. 3, 387-393.

7. H. Yilmaz Atay (2020). Drug Deliv. Biomed. Appl., 457-489.

8. I. M. El-Sherbiny and E. Salih (2018). Green Met. Nanopart., 293-319.

9. N. Garcia, Y. H. Kao, and M. Strongin (1972). Reg. Phys. Revis. 5, 2029-2039.

10. F. Dong, T. Xiong, Y. Sun, Z. Zhao, Y. Zhou, X. Feng, and Z. Wu (2014). Chem. Commun. 50, 10386-10389.

11. F. Xia, X. Xu, X. Li, L. Zhang, L. Zhang, H. Qiu, W. Wang, Y. Liu, and J. Gao (2014). Ind. Eng. Chem. Resour. 53, 10576-10582.

12. F. Qin, R. Wang, G. Li, F. Tian, H. Zhao, and R. Chen (2013). Catal. Commun. 42, 14-19.

13. H. Safardoust-Hojaghan, M. Salavati-Niasari, M. H. Motaghedifard, and S. M. Hosseinpour-Mashkani (2015). N. J. Chem. 39 (6), 4676-4684.

14. F. Wang, R. Tang, H. Yu, P. C. Gibbons, and W. E. Buhro (2008). Chem Mater. 20, 3656-3662.

15. Y. Xu, Z. Ren, W. Ren, G. Cao, K. Deng and Y. Zhong (2008). Nanotechnology, 19(11), 115602.

16. M. Gulrajani, D. Gupta, S. Periyasamy, and S. Muthu (2008). J. Appl. Poly Sci. 108, 614-623.

17. J. Li, H. Q. Fan, J. Chen, and J. L. Liu (2009). Colloids Surf. A 340, 66-69.

18. Z. Wang, R. Su, D. Wang, J. Shi, J.-X. Wang, Y. Pu, and J.-F. Chen (2017). Ind. Eng. Chem. Res. 56 (46), 13610-13617.

19. C. Gomez, G. Hallot, A. Pastor, S. Laurent, E. Brun, C. SicardRoselli, and M. Port (2019). Ultrason. Sonochem 56, 167-173.

20. L. S. Rieznichenko, T. G. Gruzina, S. M. Dybkova, V. O. Ushkalov, and Z. R. Ulberg (2015). Am. J. Bioterror. Biosecur. Biodefens 2 (1), 1004.

21. Y. Liang, J. Manioudakis, J. Macairan, M. S. Askari, P. Forgione, and R. Naccache (2019). ACS Omega 4 (12), 14955-14961.

22. M. Elshikh, S. Ahmed, S. Funston, P. Dunlop, M. McGaw, R. Marchant, and I. M. Banat (2016). Biotechnol. Lett. 38 (6), 1015-1019.

23. R. Hernandez-Delgadillo, D. Velasco-Arias, D. Diaz, K. ArevaloNino, M. Garza-Enriquez, M. A. D. Garza-Ramos, and C. CabralRomero (2012). Int. J. Nanomedicine 7, 2109-2113.

24. P. E. Das, A. F. Majdalawieh, A. I. Abu-Yousef, S. Narasimhan, and P. Poltronieri (2020). Materials (Basel) 13 (4), 876.

25. A. B. Sieval, M. Thanoual, A. F. Kotze, J. C. Verhoef, J. Brussee and H. E. Junginger (1998). Carbohydr. Polym. 36, 157-165 (1998)

26. S. Adewuyi, N. O. Sanyaolu, S. A. Amolegbe, A. O. Sobola, and O. M. Folarin (2012). J. Environ. Sci. 24 (9), 1702-1708.

27. D. Ma, J. Zhao, Y. Zhao, X. Hao, L. Li, L. Zhang, Y. Lu, and C. Yu (2012). Colloids Surf. A 395, 276-283.

28. J. A. Creighton and D. G. Eadon (1991). J. Chem. Soc. Faraday Trans. 87, 3881-4389.

29. Y. Ding, X.-H. Xia, and C. Zhang (2006). Nanotechnology 17 4156-4162.

30. V. Mishra, V. Baranwal, R. K. Mishra, S. Sharma, B. Paul, and A. C. Pandey (2017). Sci. Rep. 7 (1), 18032.

31. L. Escobar-Alarcón, J. G. Morales-Mendez, D. A. Solís-Casados, S. Romero, M. Fernández and E. Haro-Poniatowski (2015). J. Phys. 582, 012013. 
32. Joint Committee for Powder Diffraction Standards (JCPDS). Power diffraction file for inorganic materials (1979).

33. Y. N. Slavin, J. Asnis, U. O. Häfeli, and H. Bach (2017). J. Nanobiotechnol. 15, 65.

34. S. Saha, P. A. Anjali, S. Kundu, S. Basu, and T. Pal (2010). Langmuir 26, 885-2893.

35. A. Gangula, R. Podila, M. Ramakrishna, L. Karanam, C. Janardhana, and A. M. Rao (2011). Langmuir 27, 15268-15274.

36. S. Liu, A. Qileng, J. Huang, Q. Gao, and Y. Liu (2017). RSC Adv. 7, 45545 .
37. N. Sahiner, S. Butun, O. Ozay, and B. J. Dibek (2012). Colloid. Interface Sci. 373, 122-128.

38. H. Guo, Y. Ren, Q. Cheng, D. Wang, and D. Y. Liu (2017). Catal. Commun. 102, 136-140.

Publisher's Note Springer Nature remains neutral with regard to jurisdictional claims in published maps and institutional affiliations. 